\title{
Systematic Study of Magnetic Interactions in Insulating Cuprates
}

\author{
Yoshiaki Mizuno, Takami Tohyama and Sadamichi Maekawa ${ }^{a}$ \\ ${ }^{a}$ Institute for Materials Research, Tohoku University, Sendai 980-8577, Japan.
}

The magnetic interactions in one-dimensional, two-dimensional (2D) and ladder cuprates are evaluated systematically by using small $\mathrm{Cu}-\mathrm{O}$ clusters. We find that the superexchange interaction $J$ between nearest neighbor $\mathrm{Cu}$ spins strongly depends on $\mathrm{Cu}-\mathrm{O}$ structure through the Madelung potential, and in 2D and ladder cuprates there is a four-spin interaction $J_{\text {cyc }}$, with magnitude of $10 \%$ of $J$. We show that $J_{\text {cyc }}$ has a strong influence on the magnetic excitation in the high-energy region of $2 \mathrm{D}$ cuprates.

A variety of insulating cuprates affords us an opportunity to study the magnetic properties of low-dimensional systems. Recent experiments for insulating cuprates have revealed interesting characteristics of magnetic interactions: The superexchange interaction between nearest neighbor $\mathrm{Cu}$ spins $J$ remarkably depends on $\mathrm{Cu}-\mathrm{O}$ network structure [1], and additional interactions such as a four-spin (4S) interaction are important for ladder [2] and two-dimensional (2D) cuprates [3]. These characteristics indicate the necessity to establish proper magnetic descriptions for the cuprates. In this paper we perform a systematic study of magnetic interactions for onedimensional (1D), 2D, and ladder cuprates theoretically.

A starting model to describe the electronic states of cuprates is the $d-p$ model, in which hopping integrals between $\mathrm{Cu} 3 d$ and $\mathrm{O} 2 p$ orbitals $\left(T_{p d}\right)$ and between $\mathrm{O} 2 p$ orbitals $\left(T_{p p}\right)$, an energylevel separation between the $\mathrm{Cu} 3 d$ and $\mathrm{O} 2 p$ orbitals $(\Delta)$, and Coulomb interactions on $\mathrm{Cu}$ and $\mathrm{O}$ sites are taken into account. $T_{p d}$ and $T_{p p}$ are obtained by considering not only the bond length dependence but also the effect of the Madelung potential around $\mathrm{Cu}$ and $\mathrm{O}$ ions. We find that the potential enhances the magnitudes of $T_{p d}$ and $T_{p p}$ in the 1D cuprates as compared with those in the 2D ones [1]. In the two-leg ladder compounds such as $\mathrm{SrCu}_{2} \mathrm{O}_{3}, T_{p p}$ along the leg of the ladder is enhanced by the Madelung potential due to adjacent two-leg ladders. These enhancements play an important role in the dependence of $J$ on the dimensionality. The $\Delta$ is determined from the difference in the Madelung potential between $\mathrm{Cu}$ and $\mathrm{O}$ sites.

The magnetic interactions are evaluated by mapping the lowest several eigenstates of small clusters $\left(\mathrm{Cu}_{2} \mathrm{O}_{7}, \mathrm{Cu}_{4} \mathrm{O}_{12}\right.$, and $\left.\mathrm{Cu}_{6} \mathrm{O}_{17}\right)$ for the $d-p$ model onto those of the corresponding Heisenberg-type model 何. For 2D systems, we take into account not only $J$, but also a diagonal interaction $J_{\text {diag }}$ and $4 \mathrm{~S}$ interaction $J_{\text {cyc }}$ in the model: $H=J \sum_{\langle i, j\rangle} \mathbf{S}_{i} \cdot \mathbf{S}_{j}+J_{\text {diag }} \sum_{\langle\langle i, j\rangle\rangle} \mathbf{S}_{i}$. $\mathbf{S}_{j}+J_{\text {cyc }} \sum_{\text {plaquette }}\left(P_{i j k l}+P_{i j k l}^{-1}\right)$, where $\mathbf{S}_{i}$ is a spin operator at site $i$, and $J_{\text {cyc }}$ is defined as the coefficient of the $4 \mathrm{~S}$ cyclic permutation operators $P_{i j k l}$ and $P_{i j k l}^{-1}$, which can be rewritten by using the two-spin interaction $\left(\mathbf{S}_{i} \cdot \mathbf{S}_{j}\right)$ and the four-spin interactions $\left(\mathbf{S}_{i} \cdot \mathbf{S}_{j}\right)\left(\mathbf{S}_{k} \cdot \mathbf{S}_{l}\right)$. For ladder systems, we distinguish between the nearest neighbor interactions along the leg $\left(J_{\text {leg }}\right)$ and along the rung ( $\left.J_{\text {rung }}\right)$ of the ladder.

The calculated results are summarized in Table 1 , where we take $\mathrm{La}_{2} \mathrm{CuO}_{4}, \mathrm{SrCu}_{2} \mathrm{O}_{3}$, and $\mathrm{Sr}_{2} \mathrm{CuO}_{3}$ as typical systems of $2 \mathrm{D}$, ladder and ID cuprates, respectively (see Refs. 1 and 5 for the parameters used in the calculations). We find that $J$ in the $1 \mathrm{D}$ cuprate is larger than that in the $2 \mathrm{D}$ one. This is caused by the enhancement of the hopping integrals in 1D cuprates as mentioned above. For 2D cuprates, we obtain $J$ to be $\sim 0.15 \mathrm{eV}$, consistent with the experimental values [1]. In addition, we find that $J_{\text {cyc }}$ is $7 \%$ of $J$, while $J_{\text {diag }}$ is zero. These results are consistent with a previous cluster calculation [5], and a re- 
Table 1

The magnetic interactions obtained by mapping the eigenstates of $\mathrm{Cu}_{2} \mathrm{O}_{7}, \mathrm{Cu}_{4} \mathrm{O}_{12}$, and $\mathrm{Cu}_{6} \mathrm{O}_{17}$ clusters for $\mathrm{Sr}_{2} \mathrm{CuO}_{3}, \mathrm{La}_{2} \mathrm{CuO}_{4}$, and $\mathrm{SrCu}_{2} \mathrm{O}_{3}$, respectively, onto the corresponding Heisenbergtype models. The numbers in parentheses denote the deviation in the last significant digit.

\begin{tabular}{cccc}
\hline Material & $J[\mathrm{eV}]$ & $J_{\text {diag }}[\mathrm{eV}]$ & $J_{\text {cyc }}[\mathrm{eV}]$ \\
\hline $\mathrm{Sr}_{2} \mathrm{CuO}_{3}$ & 0.17 & - & - \\
$\mathrm{La}_{2} \mathrm{CuO}_{4}$ & $0.146(1)$ & $0.00(0)$ & $0.011(1)$ \\
$\mathrm{SrCu}_{2} \mathrm{O}_{3}$ & $J_{\text {leg }}: 0.195(5)$ & $0.003(2)$ & $0.018(2)$ \\
& $J_{\text {rung }}: 0.15(2)$ & & \\
\hline
\end{tabular}

cent analysis of a multimagnon spectrum [3]. For ladder cuprates, we obtain $J_{\text {leg }} / J_{\text {rung }}=1.3$. The enhancement of $T_{p p}$ along the leg of the ladder is the origin of the relation $J_{\text {leg }}>J_{\text {rung. }} J_{\text {cyc }}$ is $10 \%$ of $J_{\text {leg. }}$. Note that the ratio $J_{\text {leg }} / J_{\text {rung }}$ is smaller than that believed so far, i.e., $J_{\text {leg }} / J_{\text {rumg }} \sim 2$ [6]. However, the Heisenberg ladder with the present values of $J_{\text {leg }}, J_{\text {rung }}, J_{\text {cyc }}$ and $J_{\text {diag }}$ reproduces very well the experimental results of the temperature dependence of the magnetic susceptibility (not shown here). Therefore, we consider the values shown in Table 1 to be reasonable. Here we would like to emphasize that $J_{\text {cyc }}$ plays a crucial role in obtaining the good agreement between the experimental and theoretical magnetic susceptibilities [4].

Next, in order to examine the effect of $J_{\text {cyc }}$ on the magnetic excitation, we calculate the dynamical spin-correlation function $S(\mathbf{q}, \omega)$ for $2 \mathrm{D}$ cuprates. Figure 1 shows the dispersion and the intensity of $S(\mathbf{q}, \omega)$ for a $4 \times 4$ Heisenberg model with $J=0.146 \mathrm{eV}$ and $J_{\text {cyc }}=0.011 \mathrm{eV}$. For comparison, the result for $J_{\text {cyc }}=0$ are also shown. We find that the intensity is not sensitive to $J_{\text {cyc }}$, while the dispersion is strongly suppressed in the highenergy region. In particular, it is worth noting that $\omega(\mathbf{q})$ at $\mathbf{q}=(\pi / 2, \pi / 2)$ becomes smaller than that at $\mathbf{q}=(\pi, 0)$. This is in contrast with the case of $J_{\text {cyc }}=0$, in which the magnetic zone boundary $(\mathbf{q}=(\pi, 0) \rightarrow(\pi / 2, \pi / 2))$ has a flat dispersion. Therefore, it is desirable that inelastic neutronscattering experiments in the wide energy region

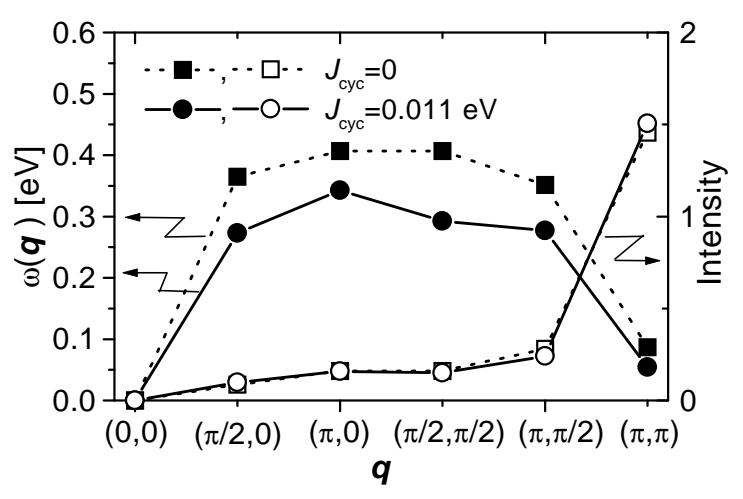

Figure 1. The magnetic excitation spectra of a $4 \times 4$ Heisenberg model with $J$ and $J_{\text {cyc }}$.

be performed to verify the role of $J_{\text {cyc }}$, that is, the suppression of the dispersion at $(\pi / 2, \pi / 2)$.

In summary, we have evaluated the magnetic interactions in various cuprates systematically. We have shown that an ionic nature inherent in insulating cuprates is important for the material dependence of $J$. We found that $J_{\text {cyc }}$ is $\sim 10 \%$ of $J$, and greatly influences the magnetic excitation spectra in $2 \mathrm{D}$ cuprates.

This work was supported by a Grant-in-Aid for Scientific Research on Priority Areas from the Ministry of Education, Science, Sports and Culture of Japan, CREST and NEDO. The parts of the numerical calculation were performed in the Supercomputer Center in ISSP, University. of Tokyo, and the supercomputing facilities in IMR, Tohoku University.

\section{REFERENCES}

1. Y. Mizuno, T. Tohyama and S. Maekawa, Phys. Rev. B 58, R14713 (1999).

2. M. Matsuda, K. Katsumata, R. S. Eccleston, S. Brehmer and H.-J. Mikeska, unpublished.

3. J. Lorenzana, J. Eroles and S. Sorella, Phys. Rev. Lett. 83, 5122 (1999).

4. Y. Mizuno, T. Tohyama and S. Maekawa, J. Low Temp. Phys. 117389 (1999).

5. H. J. Schmidt and Y. Kuramoto, Physica C 167, 263 (1990).

6. J.C.Johnston, Phys. Rev. B 54, 13009 (1996). 